\title{
The role of dasatinib in the management of chronic myeloid leukemia
}

This article was published in the following Dove Press journal:

Drug Design, Development and Therapy

9 February 2015

Number of times this article has been viewed

\section{Runzhe Chen \\ Baoan Chen}

Department of Hematology and Oncology (Key Department of Jiangsu Medicine), Zhongda Hospital, Medical School, Southeast University, Nanjing, Jiangsu Province, People's Republic of China
Correspondence: Baoan Chen Department of Hematology and Oncology, Zhongda Hospital, Medical School, Southeast University, Dingjiaqiao 87, Gulou District, Nanjing, Jiangsu Province, People's Republic of China Tel +86 25 83272006

Fax +86258327 20II

Email cba8888@hotmail.com
Abstract: Dasatinib is a second-generation tyrosine kinase inhibitor (TKI) for chronic, blastic, or accelerated phase chronic myeloid leukemia (CML) patients who are resistant or intolerant to previous treatment. It potently inhibits BCR/ABL and SRC-family kinases (SRC, LCK, HCK, YES, FYN, FGR, BLK, LYN, FRK), as well as c-KIT, PDGFR-a and -b, and ephrin receptor kinase. Various clinical trials have provided evidence that it has more durable complete hematologic and cytogenetic responses, as well as more potency in imatinib-resistant or -intolerant CML, and it has also shown its advantages in newly diagnosed CML compared to imatinib. In this review, we mainly focus on the structure, mechanisms, pharmacokinetics, and pharmacogenetics of dasatinib. We also summarize clinical trials with dasatinib on CML and provide our recommendations for dasatinib in the treatment of CML.

Keywords: imatinib, tyrosine kinase inhibitor, clinical trials

\section{Introduction}

Chronic myeloid leukemia (CML) is a malignant hematopoietic stem cell disorder with an incidence of 1-2 cases per 100,000 adults. It is characterized by cells carrying a Philadelphia $(\mathrm{Ph})$ chromosome, forming by the $B C R-A B L$ oncogene, which was a fusion of the Abelson oncogene $(A B L)$ from chromosome $9 \mathrm{q} 34$ and the breakpoint cluster region $(B C R)$ gene on $22 \mathrm{q} 11.2 .{ }^{1,2}$ The $B C R-A B L$ gene results in a deregulated kinase activity that promotes growth and replication through downstream pathways such as RAS, RAF, JUN kinase, MYC, and STAT, leading to impaired apoptosis and uncontrolled proliferation. ${ }^{3-5}$ Deregulating BCR-ABL tyrosine kinase (TK) activity provides an identifiable target that has considerably revolutionized the treatment of CML and changed the management and outcome of CML patients, with a survival rate of $88 \%-95 \%$ reported at 6 years. ${ }^{6-9}$ The first-generation of BCR-ABL tyrosine kinase inhibitor (TKI) is imatinib mesylate, and it is the frontline therapy for CML; however, in newly diagnosed patients with chronic phase CML, the rate of resistance to imatinib at 4 years was up to $20 \%$, increasing to $70 \%-90 \%$ for patients in the accelerated/blastic phase.

Resistance to imatinib led to the development of novel TKIs. ${ }^{10,11}$ Dasatinib (Sprycel $^{\circledR}$, Bristol-Myers Squibb, New York, NY, USA), a second-generation TKI, was approved by the US Food and Drug Administration (FDA) in 2010 at $100 \mathrm{mg}$ once daily as the starting dose in patients with chronic phase CML and at $70 \mathrm{mg}$ twice daily in patients for the treatment of chronic, blastic, or accelerated phase CML resistant or intolerant to previous treatment, including imatinib. ${ }^{11-13}$ Various clinical trials have provided evidence that it has more durable complete hematologic and cytogenetic responses and more potency (325 times more active) compared to imatinib. ${ }^{11,14-16}$ In this review, we mainly focus on addressing the structure, mechanisms, pharmacokinetics, 
and pharmacogenetics of dasatinib. We also summarize clinical experiences with dasatinib on CML and provide our recommendations for critical strategies of dasatinib in the treatment of CML.

\section{Structure, mechanisms, pharmacokinetics, and pharmacogenetics}

\section{Structure of dasatinib}

Dasatinib (Figure 1) is a multitargeted kinase inhibitor that was discovered by and named after Jagabandhu Das. It potently inhibits $\mathrm{BCR} / \mathrm{ABL}$ and the $\mathrm{SRC}$ family kinases (SRC, LCK, HCK, YES, FYN, FGR, BLK, LYN, FRK), as well as c-KIT, PDGFR-a and -b, and ephrin receptor kinase. Clinically more significant, dasatinib has been shown to block $\mathrm{G}_{1} / \mathrm{S}$ transition and inhibit cell growth and BCR-ABL with greater potency compared to other BCR-ABL inhibitors. ${ }^{4,17}$

\section{The mechanism of dasatinib for CML}

The concrete mechanism behind dasatinib is still unknown and there are many theories. It may cause immunological modifications that can display more diverse $\mathrm{T}$ cell populations compared with imatinib; this could be confirmed by having a higher proportion of effector $\mathrm{CD}^{+} \mathrm{T}$ cells in dasatinib-treated CML patients. ${ }^{2,18}$ Increases in dendritic cells, cytotoxic $\mathrm{T}$ and natural killer cells in peripheral blood samples have already been reported in cases treated by dasatinib, ${ }^{19}$ and one study confirmed that elevated serum interleukin-10 levels by activated dendritic cells could modulate the chemotherapeutic activity of dasatinib. ${ }^{20}$ Based on previous findings, dasatinib treatment may provide clinical benefit in inflammatory diseases. $^{21,22}$ However, the strength of immunomodulatory effects could also be influenced by some clinically relevant side effects such as pleural effusions and thrombocytopenia after the treatment of dasatinib. ${ }^{23,24}$

Dasatinib may also have a more profound effect on the leukemic stem and progenitor cells than imatinib, and influences the tumor microenvironment, ${ }^{4,25,26}$ but it still needs further confirmation. Some studies have also showed that dasatinib induces defects in spindle generation, cell cycle arrest, and centrosome alterations in leukemic cells, tumor cell lines, as well as in normal cells. ${ }^{27}$

\section{Pharmacokinetics and pharmacogenetics}

Dasatinib can be rapidly absorbed and reach peak plasma concentrations $0.5-3$ hours after oral administration, for which the solubility is dependent on $\mathrm{pH}$. In patients with leukemia, the calculated apparent volume of distribution for dasatinib was 2,502 $\mathrm{L}$ and the estimated elimination half-life was approximately $3-5$ hours. It is extensively metabolized in the liver, predominantly by cytochrome P 450 (CYP) 3 A4. ${ }^{17}$ Optimal clinical benefit from dasatinib could only depend to a large extent on achieving optimal drug exposure of plasma concentrations. This can be affected by several factors, such as individual patient variability in absorption, distribution, and metabolism, genetic or demographic differences between individuals, and even the gastrointestinal tract. Adherence, drug-to-drug and drug-to-diet interactions or environmental influences could also impact on drug exposure. ${ }^{28}$

The pharmacogenetics of dasatinib are seldom reported; however, we think that the stratification of patients according to genotypes could be proposed for drug selection between dasatinib and other TKIs, and will represent an attractive opportunity for new clinical trials.

\section{Basic research of dasatinib in CML}

Several basic studies have been dedicated to the relationships between dasatinib and CML. Dasatinib has significant inhibitory activity against both wild-type KIT and the KIT oncoprotein (KITD816V) mutation. ${ }^{29}$ The receptor tyrosine kinase DDR1 and the oxidoreductase NQO2 have also been identified as the targets of dasatinib as discovered by Rix et al recently. ${ }^{22}$

The expansion of a mutant $\mathrm{Ph}$-positive clone could be the reason why some CML patients respond well to dasatinib

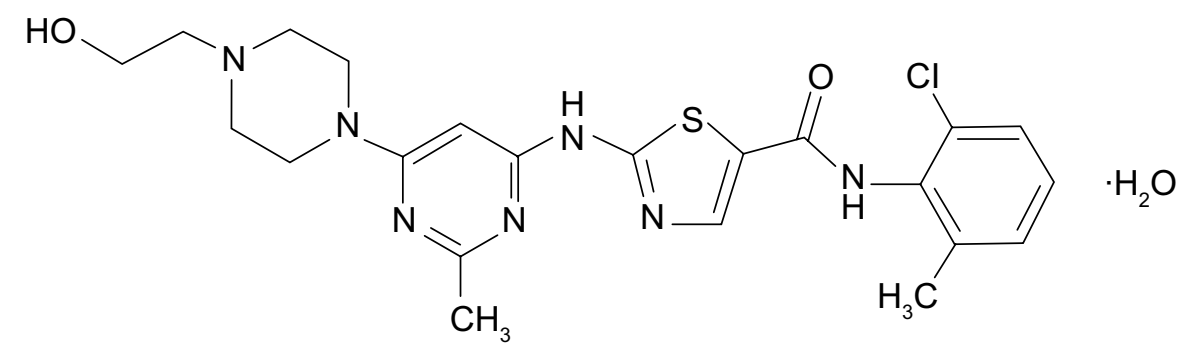

Figure I Chemical structure of dasatinib. 
after failing of imatinib. It may be due either to late acquisition of a second mutation in the originally mutated clone (eg, T315I), or to acquisition of a completely new mutant clone (eg, F317L).$^{30}$ One point that has to be mentioned is that the F317L mutation is resistant to dasatinib but sensitive to other TKIs. The prognosis is dependent mostly on the disease stage. ${ }^{31}$

What we are reluctant to see is that ongoing TKI like dasatinib exposure might alter the patterns of recurrence and favor the outgrowth of clones with TKI-resistant mutations. ${ }^{32} \mathrm{~A}$ high frequency of polymutant BCR-ABL1 alleles is involved in resistance to dasatinib in CML and may unveil unique mechanisms of escape of dasatinib therapy to $\mathrm{CML} ;{ }^{33}$ however, the disease phase has nothing to do with dasatinib failure. ${ }^{34}$ Silveira et $\mathrm{al}^{35}$ found that long non-coding RNAs (InRNAs) are implicated in the pathway, leading to tumorigenesis, and may be involved in dasatinib resistance in CML. Skorski ${ }^{36}$ suggested that CML refractory or resistant to TKIs was associated with leukemia stem cells (LSCs) and/or leukemia progenitor cells (LPCs) and eventually generates additional TKI-resistant clones with complex karyotypes.

In order to overcome the CML resistance of TKIs, many scientists are working hard to find new ways and strategies. $\mathrm{Bu}$ et $\mathrm{al}^{37}$ discovered that the histone deacetylase inhibitor (HDACi) suberoylanilide hydroxamic acid (SAHA) could exert synergistically with a novel combination regimen S116836 to promote apoptosis in the CML, including those resistant to imatinib or dasatinib. Chen et $\mathrm{a}^{38}$ discovered that treatment with omacetaxine mepesuccinate can kill more than $90 \%$ of the LSCs with BCR-ABL-induced CML, while less than $9 \%$ or $25 \%$ of the LSCs were killed after treating with imatinib or dasatinib. The activity of omacetaxine in CML seems to be independent of the BCR-ABL mutation status and it can be found in dasatinib-resistant CML. ${ }^{38}$

\section{Clinical trials of dasatinib in CML}

The efficacy of dasatinib in the treatment of CML has been investigated in several clinical trials, including various Phase I, II, and III studies on register.

\section{Dasatinib in patients with imatinib- resistant or -intolerant CML}

For clinical trials on dasatinib in the treatment of imatinibresistant or -intolerant CML, dasatinib produced hematologic and cytogenetic responses in a significant number of patients.

A Phase I study performed by Talpaz et al ${ }^{39}$ enrolled patients at various phases of $\mathrm{CML}$ or with $\mathrm{Ph}^{+}$acute lymphoblastic leukemia (ALL) who could not tolerate or were resistant to imatinib. Dasatinib (15-240 mg per day) was administered in 4-week treatment cycles, once or twice daily. A complete hematologic response (CHR) was seen in 37 of 40 patients with chronic-phase CML, and major hematologic responses (MaHRs) were achieved in 31 of 44 patients with accelerated-phase CML, CML with blast crisis, and $\mathrm{Ph}^{+} \mathrm{ALL}$. In these two phases, the rates of major cytogenetic responses (MCyRs) were 45\% and 25\%, respectively. Responses were maintained in $95 \%$ of patients with chronic-phase disease and in $82 \%$ of patients with accelerated-phase disease, with a median follow-up of more than 12 months and 5 months, respectively. Nearly all patients with lymphoid blast crisis (LBC) and $\mathrm{Ph}^{+} \mathrm{ALL}$ had a relapse within 6 months. From a Phase I/II clinical study, Sakamaki et $\mathrm{al}^{40}$ reported their results in Japanese patients, demonstrating that dasatinib was promising as a new treatment for Japanese patients with CML. Another Phase I study was conducted only for children and adolescents with relapsed or refractory leukemia. Compared to CML in adults, either $60 \mathrm{mg} / \mathrm{m}^{2}$ or $80 \mathrm{mg} / \mathrm{m}^{2}$ of the dasatinib dosage for children can be selected, but further studies were required. ${ }^{41}$

Cortes et $\mathrm{l}^{15}$ reported the results of their Phase II clinical trial of dasatinib in patients with imatinib-resistant or -intolerant blast crisis CML (myeloid blast crisis [MBC], $\mathrm{n}=74$; LBC, $\mathrm{n}=42$ ). At the 8-month follow-up, dasatinib induced MaHRs in $34 \%$ and $31 \%$ of MBC- and LBC-CML patients and MCyRs in $31 \%$ and $50 \%$ of these patients, respectively. Most $(86 \%)$ of these MCyRs were complete cytogenetic responses (CCyRs). Cytopenias were common and could be managed by dose modification. In another Phase II study, Guilhot et $\mathrm{al}^{16}$ showed their results of dasatinib only with CML in the accelerated phase (CML-AP). At 8 months' minimum follow-up, $81 \%, 64 \%$, and $39 \%$ of patients achieved overall response, MaHRs, and CHRs, respectively, whereas 33\% and $24 \%$ attained major and complete cytogenetic remission. Seventy-six percent of patients were estimated to be alive and progression-free at 10 months. Response rates for the $60 \%$ of patients with baseline BCR-ABL mutations did not differ from the total population. From the follow-up of this study, the 12-month progression-free survival and overall survival rates were $66 \%$ and $82 \%$, respectively. ${ }^{42}$ Hochhaus et $\mathrm{al}^{43}$ also conducted a Phase II study of 186 patients with chronicphase CML (CML-CP) and evaluated the efficacy and safety of dasatinib (70 mg twice daily). They found that dasatinib induced notable responses, with $90 \%$ and $52 \%$ of patients achieving CHR and MCyR, respectively. After their extended follow-up of this trial, they again confirmed that dasatinib 
was associated with high response rates in patients with imatinib-resistant or -intolerant CML-CP. ${ }^{44}$ Another Phase II study in CML-CP conducted by Kantarjian et $\mathrm{al}^{45}$ randomized patients with CML-CP 2:1 to $140 \mathrm{mg}$ dasatinib ( $\mathrm{n}=101)$ or $800 \mathrm{mg}$ imatinib $(\mathrm{n}=49)$, and after a median follow-up of 15 months, CHRs were observed in $93 \%$ and $82 \%$ of patients receiving dasatinib and high-dose imatinib, respectively; thus, dasatinib resulted in higher MCyRs (52\%) than highdose imatinib (33\%). In their 2-year follow-up of this trial, dasatinib demonstrated durable responses and improved response and progression-free survival rates. ${ }^{46}$ From the interim results of a Phase II study, Ottmann et $\mathrm{al}^{47}$ confirmed that dasatinib induces rapid hematologic and cytogenetic responses in adult patients with $\mathrm{Ph}^{+} \mathrm{ALL}$ with resistance or intolerance to imatinib.

In an open-label Phase III trial, 670 patients with imatinib-resistant or -intolerant CML-CP were randomly assigned 1:1:1:1 between four dasatinib treatment groups: $100 \mathrm{mg}$ once daily, $50 \mathrm{mg}$ twice daily, $140 \mathrm{mg}$ once daily, or $70 \mathrm{mg}$ twice daily, and with a minimum follow-up of 6 months, dasatinib $100 \mathrm{mg}$ once daily was discovered to retain the efficacy of $70 \mathrm{mg}$ twice daily with less toxicity. ${ }^{48}$ In Shah et al's follow-up study, they found that imatinibresistant or -intolerant patients with CML-CP could experience long-term benefit with dasatinib therapy, particularly if achieving BCR-ABL no more than $10 \%$ at 3 months. ${ }^{48}$ In another Phase III study, Kantarjian et $\mathrm{al}^{49}$ compared the efficacy and safety of dasatinib $140 \mathrm{mg}$ once daily with the current twice-daily regimen and reported their results from the subgroup with CML-AP with a median follow-up of 15 months, and their results demonstrated that dasatinib $140 \mathrm{mg}$ once daily has similar efficacy to dasatinib $70 \mathrm{mg}$ twice daily but with an improved safety profile, Saglio et al ${ }^{50}$ also confirmed the same results in blast phase CML. However, in a Phase III study conducted by Lilly et al ${ }^{51}$ dasatinib $140 \mathrm{mg}$ once daily showed similar overall efficacy and safety in patients with imatinib-resistant or -intolerant $\mathrm{Ph}^{+} \mathrm{ALL}$.

\section{Clinical trials on dasatinib versus imatinib with newly diagnosed CML}

Two hundred and fifty-three patients with newly diagnosed CML-CP were randomized to imatinib $400 \mathrm{mg} /$ day or dasatinib $100 \mathrm{mg} /$ day in a study conducted by Radich et al. ${ }^{52}$ They discovered that the proportion of patients achieving a complete cytogenetic remission rate was superior with dasatinib ( $84 \%$ vs $69 \%$ ), as was the 12 -month molecular response $(\mathrm{MR})$ by the proportions of patients achieving $>3$ $\log ,>4-\log$, and $>4.5-\log$ reductions in BCR-ABL transcript levels. Among patients who achieved CHR, 3-year relapsefree survival was $91 \%$ with dasatinib and $88 \%$ with imatinib $400 \mathrm{mg}$. Thus, dasatinib induced more CCyR and deeper MRs after 12 months.

Kantarjian et $\mathrm{al}^{53}$ assessed the efficacy and safety of dasatinib, as compared with imatinib, for the first-line treatment of CML-CP. Five hundred and nineteen patients with newly diagnosed CML-CP were randomly assigned to receive dasatinib at a dose of $100 \mathrm{mg}$ once daily (259 patients) or imatinib at a dose of $400 \mathrm{mg}$ once daily (260 patients). After a minimum follow-up of 12 months, the rate of confirmed CCyR was higher with dasatinib than with imatinib. The safety profiles of the two treatments were similar. As a result, dasatinib may improve long-term outcomes among patients with newly diagnosed CML-CP. The 2-year follow-up by the same researchers demonstrated that dasatinib continues to show faster and deeper responses compared with imatinib. ${ }^{54}$ Dasatinib $100 \mathrm{mg}$ once daily has demonstrated efficacy in both the East Asian subpopulation and Japanese population after follow-up..$^{5,56}$ The same results were also confirmed by a study conducted by Hjorth-Hansen et al. ${ }^{57}$

\section{Side effects of dasatinib treatment}

Dasatinib was generally well-tolerated by the patients reported in the clinical trials above; ${ }^{41,44,58-61}$ however, side effects also occurred in a small group of patients. According to the results of clinical studies, the most common non-hematologic adverse events were gastrointestinal disorders such as abdominal pain, diarrhea, nausea, vomiting, gastrointestinal bleeding, mucosal inflammation, abdominal distension, and dyspepsia. Respiratory disorders such as pleural effusion, dyspnea, cough, and even pulmonary arterial hypertension were less common. Nervous system disorders including headache and neuropathy were also less common. Cardiac adverse events such as left ventricular dysfunction, cardiac failure, cardiomyopathy, ventricular failure, diastolic dysfunction, and QT-interval prolongation were less infrequent. Other toxicities such as skin sequelae, severe infections, irregular menses, vertigo, appetite disturbances and hemorrhage were reported. Management of these toxicities could be dose reduction, interruption, or discontinuation.

\section{Comparison with other second- generation TKIs}

Other second-generation TKIs include nilotinib, bosutinib, and INNO-406. Several studies compared the efficacy of dasatinib and nilotinib. Signorovitch et al ${ }^{62}$ compared MR in patients taking nilotinib $300 \mathrm{mg}$ twice daily with that in 
those taking dasatinib $100 \mathrm{mg}$ once daily by 12 months and through 48 months. The results suggested that nilotinib is associated with higher rates of achieving MR by 12 months compared to dasatinib for the treatment of newly diagnosed CML-CP and higher rates of MR achievement with nilotinib were also maintained through 48 months of followup. ${ }^{62}$ In another study, dasatinib treatment after nilotinib failure analyses showed that the ranking of strategies was mostly influenced by the duration of first- and second-line therapies. ${ }^{63}$

\section{Other choices after dasatinib failure}

There are other choices after the failure of dasatinib treatment. Changing to other TKIs could be an option. The study of ponatinib (AP24534), a third-generation TKI, which blocks both native and mutated $\mathrm{BCR}-\mathrm{ABL}$, has shown promise in recent years. ${ }^{64}$ Allogeneic hematopoietic stem cell transplantation could also be offered for those patients presenting with blast phase at diagnosis, T315I and other BCR-ABL1 mutations that are resistant or intolerant to all TKIs. ${ }^{11,65}$

\section{Cost-effectiveness of dasatinib}

A few studies were performed to assess the cost-effectiveness of dasatinib versus other treatment in CML. In a retrospective study in Sweden, Ghatnekar et $\mathrm{al}^{66}$ compared the cost of dasatinib and high-dose imatinib treatment in CML-CP patients, and found that the incremental societal cost amounts to $€ 4,250$ during the lifetime period for one person. Another study compared CML patients treated with nilotinib and dasatinib; nilotinib patients were found to be more adherent and experienced lower health care resource utilization, so nilotinib is more cost-effective in medical service cost savings compared to in dasatinib patients. ${ }^{67}$ As expected, Hoyle et $\mathrm{a}^{68}$ showed that the cost-effectiveness of dasatinib and nilotinib for imatinib-resistant individuals is uncertain for the immature clinical data. Taking into consideration the difference of each country's system and production cost, we think it is very difficult to compare the cost-effectiveness of dasatinib and other TKIs.

\section{Conclusion and future directions}

Dasatinib is an effective therapy for patients with CML. Though the mechanisms on CML are still confusing, it shows its advantages over high-dose imatinib in patients who have failed standard-dose imatinib and in those newly diagnosed CML patients. Though dasatinib is well-tolerated in most patients, side effects also occurred in a few patients. In future, the concrete mechanisms of dasatinib and its role in CML as well as how to decrease the toxicity of dasatinib require further investigation; the efficacy of dasatinib combined with other TKIs should also be evaluated in clinical trials.

\section{Acknowledgments}

This work was supported by the National Natural Science Foundation of the People's Republic of China (grant no 81170492, 81370673), National High Technology Research and Development Program 863 of People's Republic of China (grant no 2012AA022703), National Key Basic Research Program 973 of People's Republic of China (grant no 2010CB732404), Key Medical Projects of Jiangsu Province (grant no BL2014078), and Key Discipline of Jiangsu Province (2011-2015).

\section{Disclosure}

The authors report no conflicts of interest in this work.

\section{References}

1. Jabbour E, Kantarjian H. Chronic myeloid leukemia: 2014 update on diagnosis, monitoring, and management. Am J Hematol. 2014;89(5): $547-556$.

2. Kreutzman A, Ilander M, Porkka K, Vakkila J, Mustjoki S. Dasatinib promotes Th1-type responses in granzyme B expressing T-cells. Oncoimmunology. 2014;3:e28925.

3. Lindauer M, Hochhaus A. Dasatinib. Recent Results Cancer Res. 2010; 184:83-102.

4. Lindauer M, Hochhaus A. Dasatinib. Recent Results Cancer Res. 2014; 201:27-65.

5. Fava C, Rege-Cambrin G, Saglio G. Chronic myeloid leukemia: state of the art in 2012. Curr Oncol Rep. 2012;14(5):379-386.

6. Khan AM, Bixby DL. BCR-ABL inhibitors: updates in the management of patients with chronic-phase chronic myeloid leukemia. Hematology. 2014;19(5):249-258.

7. Trivedi D, Landsman-Blumberg P, Darkow T, Smith D, McMorrow D, Mullins CD. Adherence and persistence among chronic myeloid leukemia patients during second-line tyrosine kinase inhibitor treatment. J Manage Care Pharm. 2014;20(10):1006-1015.

8. Sami MB, Yousaf F, Fialho A. Severe agitation in depression precipitated by dasatinib. BMJ Case Rep. 2014;2014. pii: bcr2014204166.

9. Jain N, O'Brien $\mathrm{S}$. The frontline treatment of chronic myeloid leukemia in the chronic phase: current clinical decisions and future prospects for treatment. Expert Rev Hematol. 2013;6(5):575-586.

10. Gardner LA, Klawitter J, Gregory MA, et al. Inhibition of calcineurin combined with dasatinib has direct and indirect anti-leukemia effects against BCR-ABL1(+) leukemia. Am J Hematol. 2014;89(9):896-903.

11. Aguilera DG, Tsimberidou AM. Dasatinib in chronic myeloid leukemia: a review. Ther Clin Risk Manag. 2009;5(2):281-289.

12. Heo SK, Noh EK, Yoon DJ, Jo JC, Park JH, Kim H. Dasatinib accelerates valproic acid-induced acute myeloid leukemia cell death by regulation of differentiation capacity. PLoS One. 2014;9(2):e98859.

13. Jabbour E, Lipton JH. A critical review of trials of first-line BCR-ABL inhibitor treatment in patients with newly diagnosed chronic myeloid leukemia in chronic phase. Clin Lymphoma Myeloma Leuk. 2013;13(6): 646-656.

14. Alimena G, Breccia M, Latagliata R, et al. Dasatinib in the management of lymphoid blast crisis of Philadelphia-positive chronic myeloid leukemia with multiple extra-medullary and intracranial localizations. Leuk Res. 2009;33(8):e134-e136. 
15. Cortes J, Rousselot P, Kim DW, et al. Dasatinib induces complete hematologic and cytogenetic responses in patients with imatinib-resistant or -intolerant chronic myeloid leukemia in blast crisis. Blood. 2007; 109(8):3207-3213.

16. Guilhot F, Apperley J, Kim DW, et al. Dasatinib induces significant hematologic and cytogenetic responses in patients with imatinibresistant or -intolerant chronic myeloid leukemia in accelerated phase. Blood. 2007;109(10):4143-4150.

17. Korashy HM, Rahman AF, Kassem MG. Dasatinib. Profiles Drug Subst Excip Relat Methodol. 2014;39:205-237.

18. Nerreter T, Köchel C, Jesper D, et al. Dasatinib enhances migration of monocyte-derived dendritic cells by reducing phosphorylation of inhibitory immune receptors Siglec-9 and Siglec-3. Exp Hematol. 2014; 42(9):773-782.e1-e3.

19. Paydas S. Dasatinib, large granular lymphocytosis, and pleural effusion: useful or adverse effect? Crit Rev Oncol Hematol. 2014;89(2):242-247.

20. Futosi K, Németh T, Pick R, Vántus T, Walzog B, Mócsai A. Dasatinib inhibits proinflammatory functions of mature human neutrophils. Blood. 2012;119(21):4981-4991.

21. Sundberg TB, Choi HG, Song JH, et al. Small-molecule screening identifies inhibition of salt-inducible kinases as a therapeutic strategy to enhance immunoregulatory functions of dendritic cells. Proc Natl Acad Sci U S A. 2014;111(34):12468-12473.

22. Rix U, Hantschel O, Dürnberger G, et al. Chemical proteomic profiles of the BCR-ABL inhibitors imatinib, nilotinib, and dasatinib reveal novel kinase and nonkinase targets. Blood. 2007;110(12):4055-4063.

23. Jabbour E, Cortes J, Kantarjian H. Long-term outcomes in the secondline treatment of chronic myeloid leukemia: a review of tyrosine kinase inhibitors. Cancer. 2011;117(5):897-906.

24. Mustjoki S, Auvinen K, Kreutzman A, et al. Rapid mobilization of cytotoxic lymphocytes induced by dasatinib therapy. Leukemia. 2013; 27(4):914-924.

25. Copland M, Hamilton A, Elrick LJ, et al. Dasatinib (BMS-354825) targets an earlier progenitor population than imatinib in primary CML but does not eliminate the quiescent fraction. Blood. 2006;107(11):4532-4539.

26. Hiwase DK, Saunders VA, Nievergall E, Ross DD, White DL, Hughes TP. Dasatinib targets chronic myeloid leukemia-CD $34^{+}$progenitors as effectively as it targets mature cells. Haematologica. 2013;98(6):896-900.

27. Fabarius A, Giehl M, Rebacz B, et al. Centrosome aberrations and G1 phase arrest after in vitro and in vivo treatment with the SRC/ABL inhibitor dasatinib. Haematologica. 2008;93(8):1145-1154.

28. Widmer N, Bardin C, Chatelut E, et al. Review of therapeutic drug monitoring of anticancer drugs part two - targeted therapies. Eur J Cancer. 2014;50(12):2020-2036.

29. Shah NP, Lee FY, Luo R, Jiang Y, Donker M, Akin C. Dasatinib (BMS-354825) inhibits KITD816V, an imatinib-resistant activating mutation that triggers neoplastic growth in most patients with systemic mastocytosis. Blood. 2006;108(1):286-291.

30. Khorashad JS, Milojkovic D, Mehta P, et al. In vivo kinetics of kinase domain mutations in CML patients treated with dasatinib after failing imatinib. Blood. 2008;111(4):2378-2381.

31. Jabbour E, Kantarjian HM, Jones D, et al. Characteristics and outcome of chronic myeloid leukemia patients with F317L BCR-ABL kinase domain mutation after therapy with tyrosine kinase inhibitors. Blood. 2008;112(13):4839-4842.

32. Jones D, Thomas D, Yin CC, et al. Kinase domain point mutations in Philadelphia chromosome-positive acute lymphoblastic leukemia emerge after therapy with BCR-ABL kinase inhibitors. Cancer. 2008; 113(5):985-994.

33. Gibbons DL, Pricl S, Posocco P, et al. Molecular dynamics reveal BCR-ABL1 polymutants as a unique mechanism of resistance to PANBCR-ABL1 kinase inhibitor therapy. Proc Natl Acad Sci U S A. 2014; 111(9):3550-3555.

34. Branford S, Melo JV, Hughes TP. Selecting optimal second-line tyrosine kinase inhibitor therapy for chronic myeloid leukemia patients after imatinib failure: does the BCR-ABL mutation status really matter? Blood. 2009;114(27):5426-5435.
35. Silveira RA, Fachel AA, Moreira YB, et al. Protein-coding genes and long noncoding RNAs are differentially expressed in dasatinibtreated chronic myeloid leukemia patients with resistance to imatinib. Hematology. 2014;19(1):31-41.

36. Skorski T. Chronic myeloid leukemia cells refractory/resistant to tyrosine kinase inhibitors are genetically unstable and may cause relapse and malignant progression to the terminal disease state. Leuk Lymphoma. 2011;52 Suppl 1:23-29.

37. Bu Q, Cui L, Li J, et al. SAHA and S116836, a novel tyrosine kinase inhibitor, synergistically induce apoptosis in imatinib-resistant chronic myelogenous leukemia cells. Cancer Biol Ther. 2014;15(7):951-962.

38. Chen Y, Hu Y, Michaels S, Segal D, Brown D, Li S. Inhibitory effects of omacetaxine on leukemic stem cells and BCR-ABL-induced chronic myeloid leukemia and acute lymphoblastic leukemia in mice. Leukemia. 2009;23(8):1446-1454.

39. Talpaz M, Shah NP, Kantarjian H, et al. Dasatinib in imatinibresistant Philadelphia chromosome-positive leukemias. $N$ Engl J Med. 2006;354(24):2531-2541

40. Sakamaki H, Ishizawa K, Taniwaki M, et al. Phase $1 / 2$ clinical study of dasatinib in Japanese patients with chronic myeloid leukemia or Philadelphia chromosome-positive acute lymphoblastic leukemia. Int J Hematol. 2009;89(3):332-341.

41. Zwaan CM, Rizzari C, Mechinaud F, et al. Dasatinib in children and adolescents with relapsed or refractory leukemia: results of the CA180-018 phase I dose-escalation study of the Innovative Therapies for Children with Cancer Consortium. J Clin Oncol. 2013;31(19):2460-2468.

42. Apperley JF, Cortes JE, Kim DW, et al. Dasatinib in the treatment of chronic myeloid leukemia in accelerated phase after imatinib failure: the START a trial. J Clin Oncol. 2009;27(21):3472-3479.

43. Hochhaus A, Kantarjian HM, Baccarani M, et al. Dasatinib induces notable hematologic and cytogenetic responses in chronic-phase chronic myeloid leukemia after failure of imatinib therapy. Blood. 2007;109(6): 2303-2309.

44. Hochhaus A, Baccarani M, Deininger M, et al. Dasatinib induces durable cytogenetic responses in patients with chronic myelogenous leukemia in chronic phase with resistance or intolerance to imatinib. Leukemia. 2008;22(6):1200-1206.

45. Kantarjian HM, Pasquini R, Hamerschlak N, et al. Dasatinib or highdose imatinib for chronic-phase chronic myeloid leukemia after failure of first-line imatinib: a randomized phase 2 trial. Blood. 2007;109(12): 5143-5150

46. Kantarjian H, Pasquini R, Levy V, et al. Dasatinib or high-dose imatinib for chronic-phase chronic myeloid leukemia resistant to imatinib at a dose of 400 to 600 milligrams daily: two-year follow-up of a randomized phase 2 study (START-R). Cancer. 2009;115(18):4136-4147.

47. Ottmann O, Dombret H, Martinelli G, et al. Dasatinib induces rapid hematologic and cytogenetic responses in adult patients with Philadelphia chromosome positive acute lymphoblastic leukemia with resistance or intolerance to imatinib: interim results of a phase 2 study. Blood. 2007;110(7):2309-2315.

48. Shah NP, Kantarjian HM, Kim DW, et al. Intermittent target inhibition with dasatinib $100 \mathrm{mg}$ once daily preserves efficacy and improves tolerability in imatinib-resistant and -intolerant chronic-phase chronic myeloid leukemia. J Clin Oncol. 2008;26(19):3204-3212.

49. Kantarjian H, Cortes J, Kim DW, et al. Phase 3 study of dasatinib $140 \mathrm{mg}$ once daily versus $70 \mathrm{mg}$ twice daily in patients with chronic myeloid leukemia in accelerated phase resistant or intolerant to imatinib: 15-month median follow-up. Blood. 2009;113(25):6322-6329.

50. Saglio G, Hochhaus A, Goh YT, et al. Dasatinib in imatinib-resistant or imatinib-intolerant chronic myeloid leukemia in blast phase after 2 years of follow-up in a phase 3 study: efficacy and tolerability of 140 milligrams once daily and 70 milligrams twice daily. Cancer. 2010;116(16):3852-3861

51. Lilly MB, Ottmann OG, Shah NP, et al. Dasatinib $140 \mathrm{mg}$ once daily versus $70 \mathrm{mg}$ twice daily in patients with Ph-positive acute lymphoblastic leukemia who failed imatinib: results from a phase 3 study. Am J Hematol. 2010;85(3):164-170. 
52. Radich JP, Kopecky KJ, Appelbaum FR, et al. A randomized trial of dasatinib $100 \mathrm{mg}$ versus imatinib $400 \mathrm{mg}$ in newly diagnosed chronicphase chronic myeloid leukemia. Blood. 2012;120(19):3898-3905.

53. Kantarjian H, Shah NP, Hochhaus A, et al. Dasatinib versus imatinib in newly diagnosed chronic-phase chronic myeloid leukemia. $N$ Engl J Med. 2010;362(24):2260-2270.

54. Kantarjian HM, Shah NP, Cortes JE, et al. Dasatinib or imatinib in newly diagnosed chronic-phase chronic myeloid leukemia: 2-year follow-up from a randomized phase 3 trial (DASISION). Blood. 2012;119(5): 1123-1129.

55. Chuah CT, Nakamae H, Shen ZX, Bradley-Garelik MB, Kim DW. Efficacy and safety of dasatinib versus imatinib in the East Asian subpopulation of the DASISION trial of newly diagnosed chronic myeloid leukemia in chronic phase. Leuk Lymphoma. 2014;55(9):2093-2100.

56. Fujisawa S, Nakamae H, Ogura M, et al. Efficacy and safety of dasatinib versus imatinib in Japanese patients with newly diagnosed chronic-phase chronic myeloid leukemia (CML-CP): subset analysis of the DASISION trial with 2-year follow-up. Int J Hematol. 2014;99(2):141-153.

57. Hjorth-Hansen H, Stenke L, Söderlund S, et al. Dasatinib induces fast and deep responses in newly diagnosed chronic myeloid leukaemia patients in chronic phase: clinical results from a randomised phase-2 study (NordCML006). Eur J Haematol. Epub 2014 Aug 1.

58. Keating GM, Lyseng-Williamson KA, McCormack PL, Keam SJ. Dasatinib: a guide to its use in chronic myeloid leukemia in the EU Bio Drugs. 2013;27(3):275-279.

59. Eghtedar A, Kantarjian H, Jabbour E, et al. Outcome after failure of second generation tyrosine kinase inhibitors treatment as first-line therapy for patients with chronic myeloid leukemia. Clin Lymphoma Myeloma Leuk. 2013;13(4):477-484.

60. Krauth MT, Herndlhofer S, Schmook MT, Mitterbauer-Hohendanner G, Schlogl E, Valent P. Extensive pleural and pericardial effusion in chronic myeloid leukemia during treatment with dasatinib at $100 \mathrm{mg}$ or $50 \mathrm{mg}$ daily. Haematologica. 2011;96(1):163-166.
61. Cortes J, Kim DW, Raffoux E, et al. Efficacy and safety of dasatinib in imatinib-resistant or -intolerant patients with chronic myeloid leukemia in blast phase. Leukemia. 2008;22(12):2176-2183.

62. Signorovitch JE, Betts KA, Reichmann WM, et al. One-year and longterm molecular response to nilotinib and dasatinib for newly diagnosed chronic myeloid leukemia: a matching-adjusted indirect comparison. Curr Med Res Opin. 2014:1-8.

63. Rochau U, Sroczynski G, Wolf D, et al. Medical decision analysis for first-line therapy of chronic myeloid leukemia. Leuk Lymphoma. 2014; 55(8):1758-1767.

64. Cortes JE, Kantarjian H, Shah NP, et al. Ponatinib in refractory Philadelphia chromosome-positive leukemias. N Eng J Med. 2012;367(22): 2075-2088.

65. Jiang H, Xu LP, Liu DH, et al. Allogeneic hematopoietic SCT in combination with tyrosine kinase inhibitor treatment compared with TKI treatment alone in CML blast crisis. Bone Marrow Transplant. 2014;49(9):1146-1154.

66. Ghatnekar O, Hjalte F, Taylor M. Cost-effectiveness of dasatinib versus high-dose imatinib in patients with Chronic Myeloid Leukemia (CML), resistant to standard dose imatinib - a Swedish model application. Acta Oncol. 2010;49(6):851-858.

67. Wu EQ, Guerin A, Yu AP, Bollu VK, Guo A, Griffin JD. Retrospective real-world comparison of medical visits, costs, and adherence between nilotinib and dasatinib in chronic myeloid leukemia. Curr Med Res Opin. 2010;26(12):2861-2869.

68. Hoyle M, Rogers G, Moxham T, Liu Z, Stein K. Cost-effectiveness of dasatinib and nilotinib for imatinib-resistant or-intolerant chronic phase chronic myeloid leukemia. Value Health. 2011;14(8):1057-1067.
Drug Design, Development and Therapy

\section{Publish your work in this journal}

Drug Design, Development and Therapy is an international, peerreviewed open-access journal that spans the spectrum of drug design and development through to clinical applications. Clinical outcomes, patient safety, and programs for the development and effective, safe, and sustained use of medicines are a feature of the journal, which

\section{Dovepress}

has also been accepted for indexing on PubMed Central. The manuscript management system is completely online and includes a very quick and fair peer-review system, which is all easy to use. Visit http://www.dovepress.com/testimonials.php to read real quotes from published authors.

Submit your manuscript here: http://www.dovepress.com/drug-design-development-and-therapy-journal 